\title{
Split hand syndrome and the split hand index in ALS patients
}

\author{
Florian Antonescu ${ }^{1,2}$, Marin Adam³, Constantin Popa ${ }^{1,2}$, Sorin Tuta ${ }^{1,2}$ \\ ${ }^{1}$ National Institute of Neurology and Neurovascular Diseases, Bucharest, Romania \\ 2"Carol Davila" University of Medicine and Pharmacy, Bucharest, Romania \\ ${ }^{3}$ MEDINST Diagnostic Center, Bucharest, Romania
}

\begin{abstract}
Our objective has been a review of the recent data available in reference to the split hand clinical sign in amyotrophic lateral sclerosis (ALS) and it's novel electrophysiological counterpart the split hand index (SHI). We have conducted an extensive search on the subject literature published over the last ten years, correlating it with our own experience. ALS patients frequently present asymmetric wasting of the hand muscles, with the abductor pollicis brevis (APB) and first dorsal interosseous (FDI) more severely affected and sparing of the medial hand. The phenomenon has been called Split Hand Syndrome and is a specific clinical marker for early ALS. More recently, the SHI has been included in the electromyographical examination, being derived from the compound muscle action potentials (CMAP) of APB, FDI and abductor digiti minim (ADM). SHI has been shown to have a good sensitivity and a specificity in differentiating ALS from mimicking disorders and it also correlates with the disease progression. We conclude that the split hand sign and its electrophysiological counterpart are useful instruments for the neurologist in making an early ALS diagnosis, especially when confounding factors are present.
\end{abstract}

Keywords: motor neuron disease, amyotrophic lateral sclerosis, split hand syndrome, split hand index, split hand plus sign

\section{INTRODUCTION}

Amyotrophic lateral sclerosis (ALS) is a neurodegenerative disease in which, despite extensive ongoing research, the diagnosis remains a clinical one, with significant assistance from electrophysiological studies, especially EMG $(1,2)$.

More than twenty years have passed since Wilbourn first described asymmetric atrophy of the hand muscles in ALS patients, with preferential wasting of the lateral group, consisting of the abductor pollicis brevis (APB) and first dorsal interosseous muscle (FDI), and relative sparing of the medial group, especially abductor digiti minimi (ADM), a phenomenon he later dubbed Split Hand Syndrome $(3,4)$.

Repeated reports show the split hand to be a specific and early clinical marker for ALS $(5,6)$. In addition to its diagnostic utility, the split hand sign has provided insights into ALS physiopathology. In EMG studies a new index has been developed, to more precisely diagnose and quantify the split hand.

\section{CLINICAL PRESENTATION}

The typical presentation is that of a patient reporting slow progressing hand inability and a sunken first interosseous space. Signs suggesting diffuse ALS may be present or not. The clinical examination reveals atrophy in the first interosseous space and of the thenar eminence, with relative sparing of the hypothenar eminence. In segmental testing, the index abduction is significantly weakened, as is pollicis abduction. The finger flexors are usually spared, including the flexor pollicis longus (FPL) $(7,8)$. The patient first complains of losing the pincer grasp, with strong grip movements awkward but feasible, later there can be a shit toward a ulnar 
type of grip in which the patient grasps the objects with the ring and little finger against his palm.

Not all ALS patient which present with hand atrophy have this dissociation. In many cases, the atrophies are equal between the lateral and medial hand, this being especially true in advanced disease and bulbar onset cases.

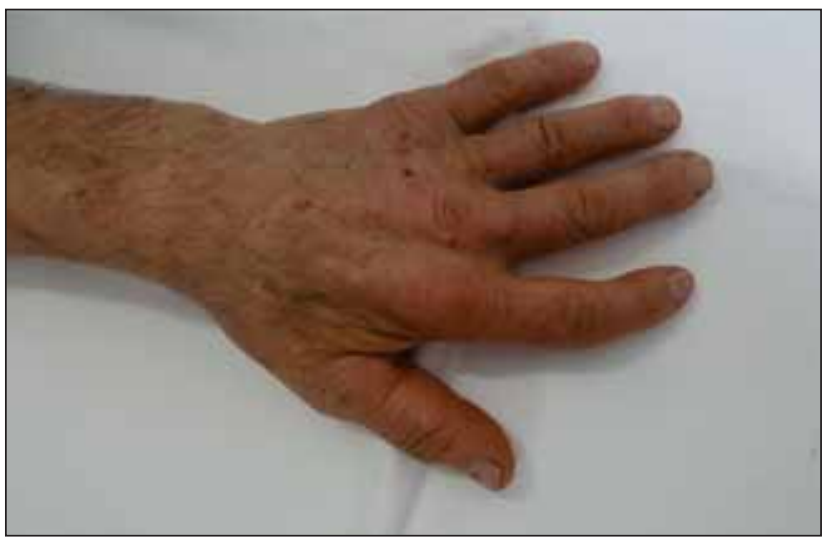

FIGURE 1. Dorsal view of the left hand in a patient with early ALS - Visible wasting of the first interosseous space, less so of the second interosseous space, last two spaces still spared

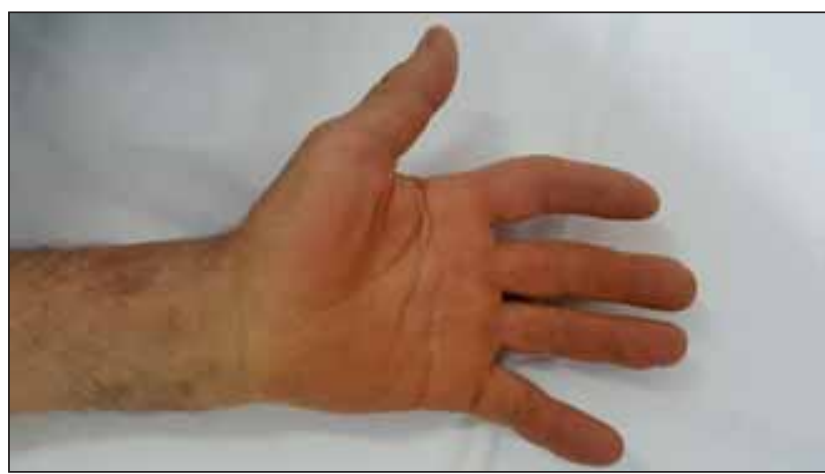

FIGURE 2. Palmar view of the same hand - Atrophy of thenar muscles with sparing of the hypothenar muscles

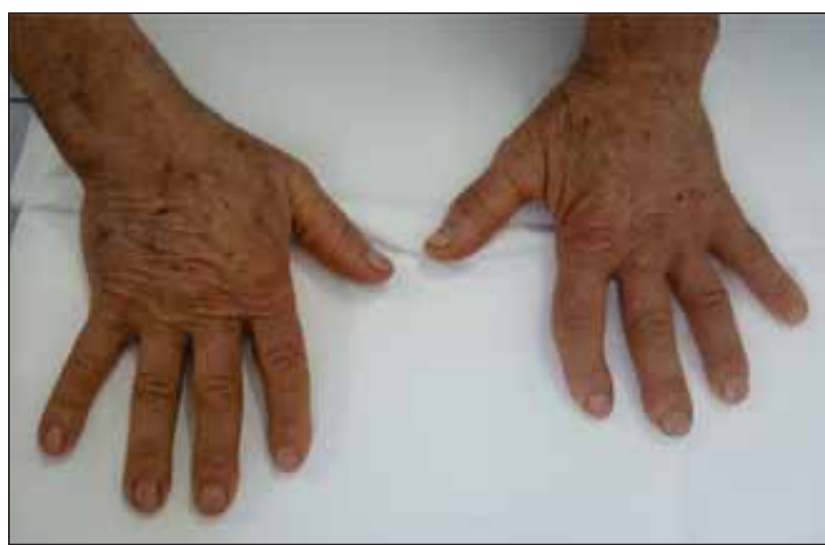

FIGURE 3. Comparison of the two hands. The left hand is the most affected limb in this patient, and the only one with significant functional loss. We see on the right incipient atrophy in the first interosseous space, compared with more significant atrophy in the first and second spaces of the left hand
The phenomenon does not occur only in ALS, being also encountered in spinal muscular atrophy, focal motor neuron disease and poliomyelitis $(4,9)$. In contrast in patients with cervical spondylotic disease the ADM and FDI, are preferentially affected, while the APB tends to be spared (9).

Recently the research concerning dissociated muscle atrophies in ALS has been expanding, with Menon describing in 2013, the split-hand plus sign, characterized by greater weakness and atrophy of the APB compared to the FPL (7). The APB and FPL muscles are innervated by the same C8-T1 motor neurons and the median nerve, the situation being similar to the ADM and FDI, both innervated from the same myotomes, through the same ulnar nerve.

\section{PHYSIOPATHOLOGY}

$\mathrm{APB}, \mathrm{FDI}$ and $\mathrm{ADM}$ are innervated by spinal motor neurons of the same segments (C8 and T1), but with different nerve pathways and, also different cortical origins. FDI and ADM are both supplied via the ulnar nerve, while APB is innervated by the median nerve.

It is not clear why the thenar muscles are preferentially affected in motor neuron disease and the mechanisms may be multifactorial (10). Several theories have been put forth, all of them centering on the fact that in humans the thumb and index finger are used much more intensely than the other fingers. This is thought to lead to greater oxidative stress and metabolic demands for upper and lower motoneurons $(9,11)$.

The corticospinal connections of APB and FDI motor neurons are much more extensive than those of ADM motor neurons, a fact also demonstrated by transcranial magnetic stimulation (TMS), and this could result in more glutamate excitotoxicity in APB and FDI spinal motor neurons $(9,10,12)$. Recently, using TMS, Menon has demonstrated a global increase in cortical excitability in ALS, but with a preferential increase in cortical excitability of the APB and FDI muscles (13).

These aspects have lead to the attractive hypothesis that the split hand phenomenon may be of cortical origin, but the evidence remain sparse. It has been also reported in autosomal dominant distal spinal muscular atrophy, spinocerebellar ataxia 
type 3 (SCA-3), and juenile muscular atrophy, entities which involve only the lower motor neuron, suggesting a peripheral origin $(14,15)$.

On the other hand membrane excitability differs between FDI and ADM motor axons even at the same location in the nerve, with nodal persistent sodium conductance, and consequently excitability, being physiologically higher in FDI axons (16). Similarly, studies have shown that excitability is physiologically higher in APB than ADM axons and that the difference increases in ALS patients $(10,17)$.

A 2017 study comparing distal motor latencies between ALS patients without spilt hand, patients with spondylotic radiculopathies and secondary hand atrophy and a control group found a more profound distal motor axonal dysfunction of the median nerve compared to the ulnar nerve even in ALS patients without split hand (18). Thus demonstrating excitability differences, between and dysfunction, at a preclinical level.

Also, studies have shown that differential atrophy of the hand occurs with a similar distribution in normal aging, probably the result of greater metabolic demands in the lateral hand region and differences in intrinsic susceptibility to oxidative stress (14). This finding raises the question if the split hand phenomenon in ALS isn't just an accelerated normal degenerative process.

\section{THE SPLIT HAND INDEX}

The differential atrophy of the hand muscles, easily observed by EMG, has, for some time, attracted the attention of electrophysiologists looking to develop from it a marker to study in relation with disease diagnosis, progression and prognosis, and the results are encouraging.

Initially the split hand phenomenon was studied electrophysiologically by comparing compound muscle action potential (CMAP) amplitudes, defining it as ADM/APB CMAP amplitude ratio $>1.7$ or ADM/FDI CMAP amplitude ratio $>1.1$. The ratio comparison was shown to have a $52 \%$ sensitivity and a $95 \%$ specificity for $\operatorname{ALS}(9,10)$.

Kuwabara used motor unit number estimation (MUNE) to study APB and ADM, noticing that in normal controls MUNE values were highest in APB, while in the ALS group the opposite was true, as the MUNE values in APB were much lower than in ADM (11).

In 2013 the Split Hand Index (SHI) was described as the result of multiplying APB CMAP with the FDI CMAP, dividing the result by the ADM CMAP (6). The intent was to incorporate all the three easily studied muscles in one single item.

It is obtained effortlessly, the examiner having only to add the FDI CMAP to the usual conduction studies, which should not prolong the examination with more than a minute.

A SHI of 5.2 was shown to have a sensitivity of $74 \%$ and a specificity of $80 \%$ in differentiating ALS from mimicking disorders (5). Compared to the previous definition based on CMAP ratios, at the mentioned cutoff value there is a $22 \%$ rise in sensibility, with a $15 \%$ loss in specificity.

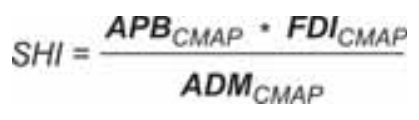

Also SHI had a significant correlation with the rate of disease progression measured through ALSFRS-R. Patients with rapid clinical progression had lower SHI values (6).

\section{CONCLUSIONS}

The split hand sign is an important clinical element in patients suspected of ALS (4), especially if the flexor pollicis longus seems to be spared (7). As the pattern may appear physiologically in old age (usually not with the severity seen in motor neuron disease), caution should be exercised not to overly alert elderly patients that don't appear to present other signs suggestive of ALS.

The differences in patterns of small hand muscle involvement can be of clinical significance in differentiating ALS from spondylotic atrophies. In spondylosis patients develop variable patterns of deficits and atrophies according to the affected spinal segments or nerve roots, but the APB is relatively spared (9).

The pathogenic mechanisms are not yet clear. There is a physiological relative hyperexcitability in the nerve fibers that supply the lateral hand muscles and an inherently intense cortical stimulations of these motor units. Both features explain an increased metabolic demand and oxidative stress, 
which seem to be reflected in a normal split hand in aging persons. On top of these, ALS adds pathological globally increased excitability, both at the cortical level and in the peripheral nerves, increasing the generation of free radicals and possibly adding glutamate mediated excitotoxicity $(19,20)$.

The split hand index is a novel EMG marker, easily assessed, that can be useful for diagnosis in

\section{REFERENCES}

1. de Carvalho M., Dengler R., Eisen A., England J.D., Kaji R., Kimura J. et al. Electrodiagnostic criteria for diagnosis of ALS. Clin Neurophysiol. 2008; 1 19(3):497-503.

2. Ludolph A., Drory V., Hardiman O., Nakano I., Ravits J., Robberecht W. et al. A revision of the El Escorial criteria - 2015 Amyotrophic Lateral Sclerosis and Frontotemporal Degeneration. 2015; 16(5-6):291-2.

3. A.J.W., P.J.S. Dissociated wasting of medial and lateral hand muscles with motor neuron disease. Can J Neurol Sci. 1994; 21(Suppl. 2 : S9).

4. Wilbourn A.J. The "split hand syndrome". Muscle Nerve [Internet]. 2000; 23(1):138. Available from: http://www.ncbi.nlm.nih.gov/ pubmed/10590421

5. Kim J.E., Hong Y.H., Lee J.H., Ahn S.W., Kim S.M., Park K.S. et al. Pattern difference of dissociated hand muscle atrophy in amyotrophic lateral sclerosis and variants. Muscle and Nerve. 2015; 51(3):333-7.

6. Menon P., Kiernan M.C., Yiannikas C., Stroud J., Vucic S. Split-hand index for the diagnosis of amyotrophic lateral sclerosis. Clin Neurophysiol [Internet]. 2013; 124(2):410-6. Available from: http://dx.doi.org/10.1016/j.clinph.2012.07.025

7. Menon P., Bae J.S., Mioshi E., Kiernan M.C., Vucic S. Split-hand plus sign in ALS: Differential involvement of the flexor pollicis longus and intrinsic hand muscles. Amyotroph Lateral Scler Front Degener. 2013; 14(4):315-8.

8. Shemesh A., Arkadir D., Gotkine M. Relative preservation of finger flexion in amyotrophic lateral sclerosis. J Neurol Sci. 2016; $361: 128-30$

9. Kuwabara S., Sonoo M., Komori T., Shimizu T., Hirashima F., Inaba A. et al. Dissociated small hand muscle atrophy in amyotrophic lateral sclerosis: Frequency, extent, and specificity. Muscle and Nerve. 2008; 37(4):426-30.

10. Shibuya K., Misawa S., Nasu S., Sekiguchi Y., Mitsuma S., Beppu M. et al. Split hand syndrome in amyotrophic lateral sclerosis: Different excitability changes in the thenar and hypothenar motor axons. J Neurol Neurosurg Psychiatry. 2013; 84(9):969-72.

11. Kuwabara S., Mizobuchi K., Ogawara K., Hattori T. Dissociated small hand muscle involvement in amyotrophic lateral sclerosis
ALS patients (21). It is most useful when the electrophysiological features are ambiguous, helping to differentiate between ALS, cervical radiculopathies, ulnar neuropathy or carpal tunnel syndrome. SHI also correlates with disease progression, especially in patients with limb onset ALS. The fact that is has been recently described continues to render it be attractive for research purposes.

Conflict of interest: none declared Financial support: none declared

detected by motor unit number estimates. Muscle and Nerve. 1999; 22(7):870-3.

12. Vucic S., Ziemann U., Eisen A., Hallett M., Kiernan M.C. Transcranial magnetic stimulation and amyotrophic lateral sclerosis: Pathophysiological insights. J Neurol Neurosurg Psychiatry. 2013; 84(10):1161-70.

13. Menon P., Kiernan M.C., Vucic S. Cortical hyperexcitability of the thenar group of intrinsic hand muscles may contribute to the split-hand sign in ALS. Clin Neurophysiol [Internet]. 2014;125(4):e1. Available from: http://dx.doi.org/10.1016/j.clinph.2013.10.023

14. Voermans N.C., Schelhaas H.J., Munneke M., Zwarts M.J. Dissociated small hand muscle atrophy in aging: The "senile hand" is a split hand. Eur J Neurol. 2006; 13(12):1381-4.

15. Schelhaas H.J., Warrenburg B.P.C. Van De, Kremer H.P.H., Zwarts M.J. The "split hand" phenomenon. 2003;2001-3.

16. Kuwabara S. S44-2 Axonal ion channel dysfunction and the split hand syndrome in ALS. Clin Neurophysiol [Internet]. 2010;121:S63. Available from: http://linkinghub.elsevier.com/retrieve/pii/ S1388245710602660

17. Shibuya K., Kanai K., Misawa S., Isose S., Noto Y., Fujimaki Y. et al. P7-11 Axonal excitability properties in patient with amyotrophic lateral sclerosis: Pathophysiology in "split hand." Clin Neurophysiol [Internet]. 2010;121:S143. Available from: http://linkinghub.elsevier. com/retrieve/pii/S1388245710605871

18. Park D., Park J.S. Terminal latency abnormality in amyotrophic lateral sclerosis without split hand syndrome. Neurol Sci. 2017; 38(5):775-81.

19. Benny R., Shetty K. Cilnical Sign :The split hand sign. Ann Indian Acad Neurol [Internet]. 2012;15(3):175. Available from: http://www. annalsofian.org/text.asp?2012/15/3/175/99700

20. Shibuya K. Dissociated small hand muscle atrophy occurs in amyotrophic lateral sclerosis: Split hand. Brain and Nerve. 2016; 68(5):501-7.

21. Menon P., Vucic S. Utility of Dissociated Intrinsic Hand Muscle Atrophy in the Diagnosis of Amyotrophic Lateral Sclerosis. J Vis Exp. 2014; (85):1-7. 